\title{
Acute Budd-Chiari syndrome
}

Dustin E Loomes MD¹, Albert Chang MD¹, Douglas Webber MD², Charles H Scudamore MD², Eric M Yoshida MD ${ }^{1}$

\section{CASE PRESENTATION}

A 23-year-old woman presented to hospital with a two-week history of abdominal pain, nausea and increasing abdominal girth. Her medical history was significant only for migraines and oral contraceptive use. Laboratory values showed an alanine aminotransferase level of $155 \mathrm{U} / \mathrm{L}$, an aspartate aminotransferase level of $85 \mathrm{U} / \mathrm{L}$, an alkaline phosphatase level of $165 \mathrm{U} / \mathrm{L}$, total bilirubin of $12 \mu \mathrm{mol} / \mathrm{L}(0.71 \mathrm{mg} / \mathrm{dL})$, an albumin concentration of $27 \mathrm{~g} / \mathrm{L}(2.7 \mathrm{~g} / \mathrm{dL})$ and an international normalized ratio of 1.2. An abdominal Doppler ultrasound and a computed tomography angiogram (Figure 1) demonstrated a nonocclusive, intrahepatic inferior vena cava (IVC) clot measuring $3.4 \mathrm{~cm} \times 1.4 \mathrm{~cm}$, as well as marked ascites.

The patient subsequently began to develop congestive hepatopathy and was scheduled for emergency placement of a portocaval shunt to protect her liver function. Caudate lobectomy was required to expose the IVC for a polytetrafluoroethylene graft anastomosis. Pathology of the caudate lobe and random biopsies from the right and left lobes showed focal hepatic vein and central venous organizing thrombosis with concomitant sinusoidal dilation (Figure 2). The patient had a relatively uncomplicated postoperative recovery. Portocaval shuntogram and pressure measurements showed a widely patent shunt with no significant pressure gradient. Thrombophilia workup was negative for any identifiable intrinsic clotting disorder.

\section{DISCUSSION}

Budd-Chiari syndrome is caused by thrombosis of the hepatic veins and/or the thrombosis of the intrahepatic or suprahepatic IVC. While an underlying etiology such as malignancy, rheumatological disorder, myeloproliferative disease, inheritable coagulopathy, infection or hyperestrogen state can be identified in many cases, the etiology remains idiopathic in $16 \%$ to $35 \%$ of cases $(1-3)$. Clinical presentations vary - ranging from acute or fulminant liver failure, to subacute (signs or symptoms for less than six months and no evidence of liver cirrhosis) to chronic (signs or symptoms for more than six months with evidence of portal hypertension and cirrhosis).

The goals of therapy are to prevent the propagation of the clot, restore patency of thrombosed veins, decompress liver congestion and manage complications such as ascites and portal hypertension. For acute thrombosis, thrombolytic therapy or radiological procedures (eg, angioplasty, stenting or transjugular intrahepatic portosystemic shunts) can be considered; however, surgical decompression remains the mainstay of treatment. Surgical shunts commonly connect the portal venous system to the IVC, creating a low-pressure route for hepatic arterial blood to drain from the liver. In patients who underwent shunt surgery before development of cirrhosis and whose shunts remain patent, five-year survival rates of as high as $89 \%$ have been reported (4-6). Chronic Budd-Chiari syndrome is managed with anticoagulation and treatment of the complications of liver failure. Rarely, liver transplantation may be necessary.
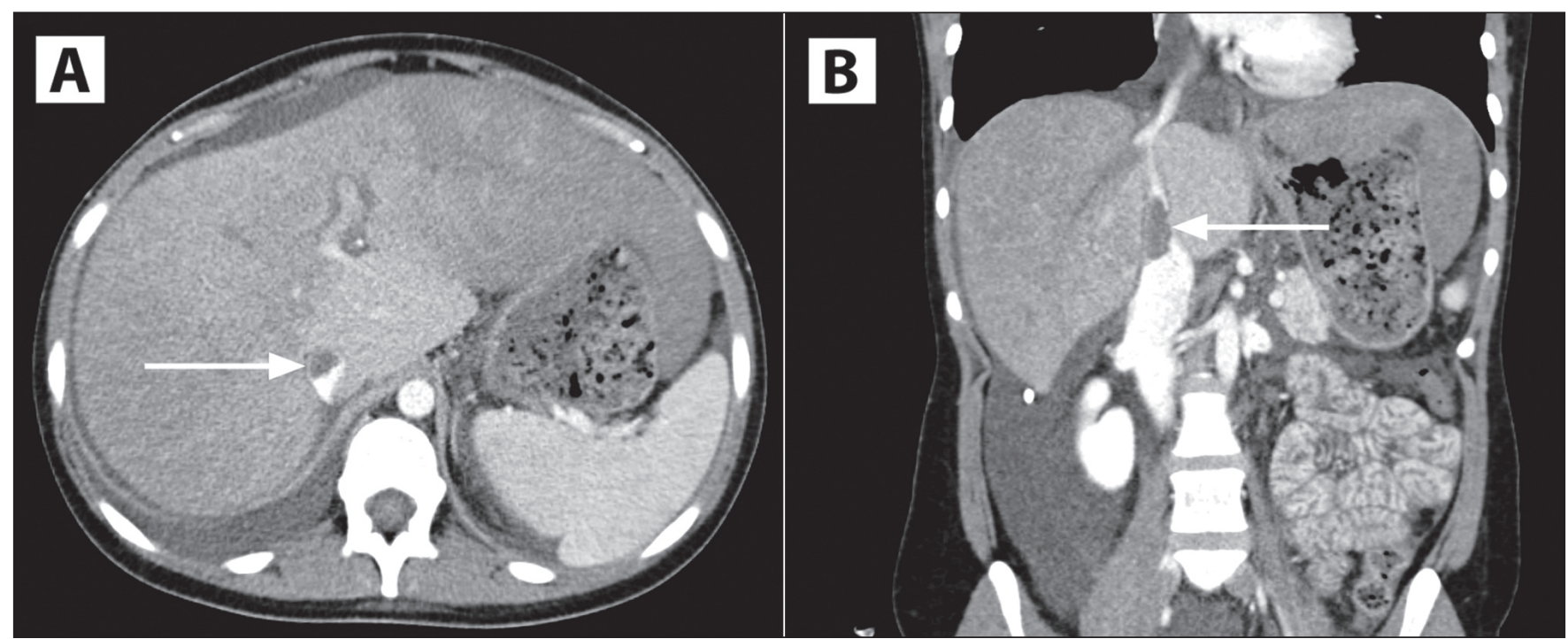

Figure 1) Axial (A) and coronal (B) views of computed tomography angiograms demonstrating a large intrahepatic inferior vena cava thrombus (arrows)

${ }^{1}$ Division of Gastroenterology; ${ }^{2}$ Department of Laboratory Medicine; ${ }^{3}$ Department of Surgery, University of British Columbia, Vancouver, British Columbia

Correspondence: Dr Eric M Yoshida, Division of Gastroenterology, Vancouver General Hospital, Diamond Health Care Centre, 5153-2775 Laurel Street,

Vancouver, British Columbia V5Z 1M9. Telephone 604-875-5371, fax 604-875-5447, e-mail eric.yoshida@vch.ca

Received for publication April 8, 2011. Accepted April 11, 2011 

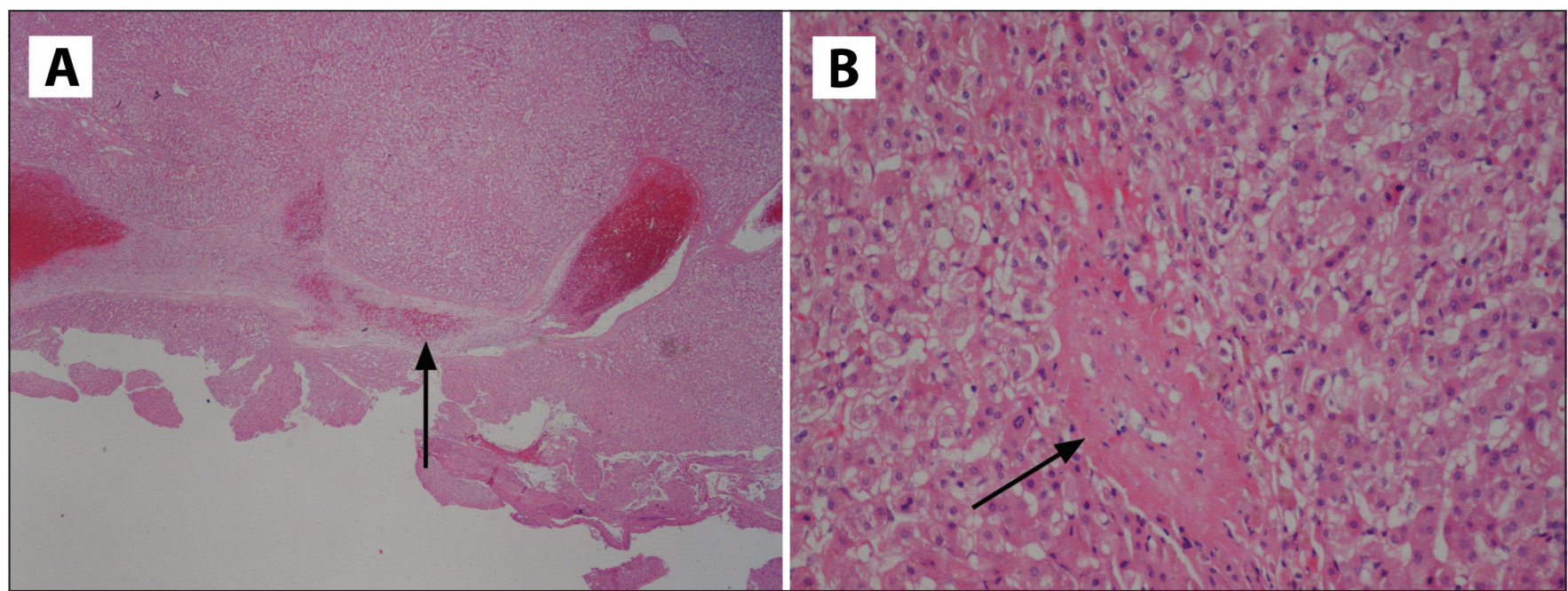

Figure 2) Hematoxylin and eosin-stained pathology sections of the caudate lobe showing organizing thrombosis (arrows) of the hepatic vein (A, original magnification $\times 20)$, and the central vein $(\mathbf{B}$, original magnification $\times 200)$

\section{REFERENCES}

1. Darwish Murad S, Plessier A, Hernandez-Guerra M, et al. Etiology, management, and outcome of the Budd-Chiari syndrome. Ann Intern Med 2009;151:167-75.

2. Dilawari JB, Bambery P, Chawla Y, et al. Hepatic outflow obstruction (Budd-Chiari syndrome). Experience with 177 patients and a review of the literature. Medicine (Baltimore) 1994;73:21-36.

3. Mitchell MC, Boitnott JK, Kaufman S, Cameron JL, Maddrey WC. Budd-Chiari syndrome: Etiology, diagnosis and management. Medicine (Baltimore) 1982;61:199-218.
4. Darwish Murad S, Valla D, De Groen P, et al. Determinants of survival and the effect of portosystemic shunting in patients with Budd-Chiari syndrome. Hepatology 2004;39:500-8.

5. Orloff MJ, Girard B. Long term results of treatment of Budd-Chiari syndrome by side to side portacaval shunt. Surg Gynecol Obstet 1989;168:33-41.

6. Panis Y, Belghiti J, Valla D, Benhamou JP, Fékété F Portosystemic shunt in Budd-Chiari syndrome: Long-term survival and factors affecting shunt patency in 25 patients in Western countries. Surgery 1994;115:276-81.

The Canadian Journal of Gastroenterology is now considering a limited number of submissions for IMAGE OF THE MONTH. These will be based on endoscopic, histological, radiological and/or patient images, which must be anonymous with no identifying features visible. The patient must consent to publication and the consent must be submitted with the manuscript. All manuscripts should be practical and relevant to clinical practice, and not simply a case report of an esoteric condition. The text should be brief, structured as CASE PRESENTATION and DISCUSSION, and not more than 700 words in length. A maximum of three images can be submitted and the number of references should not exceed five. The submission may be edited by our editorial team. 


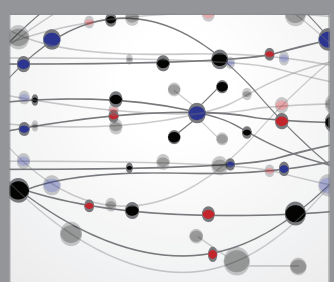

The Scientific World Journal
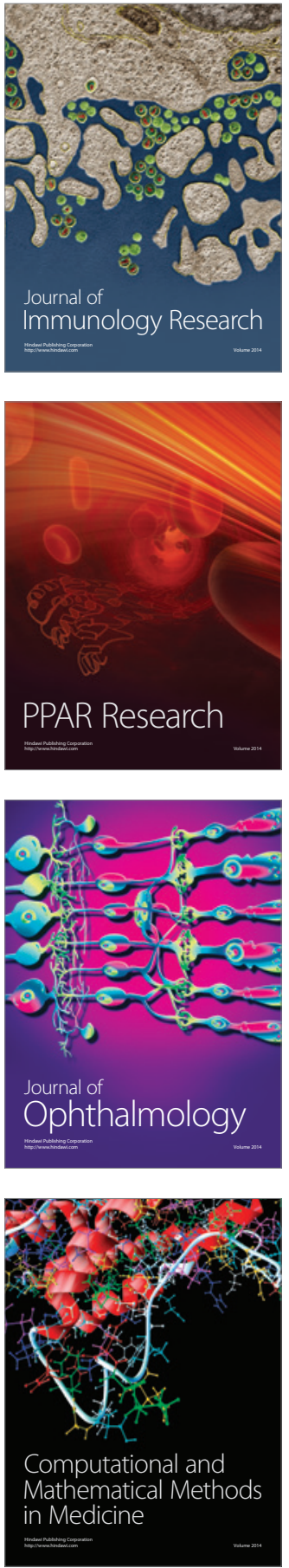

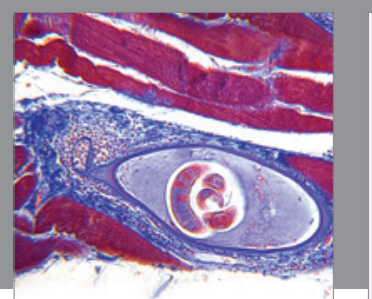

Gastroenterology Research and Practice

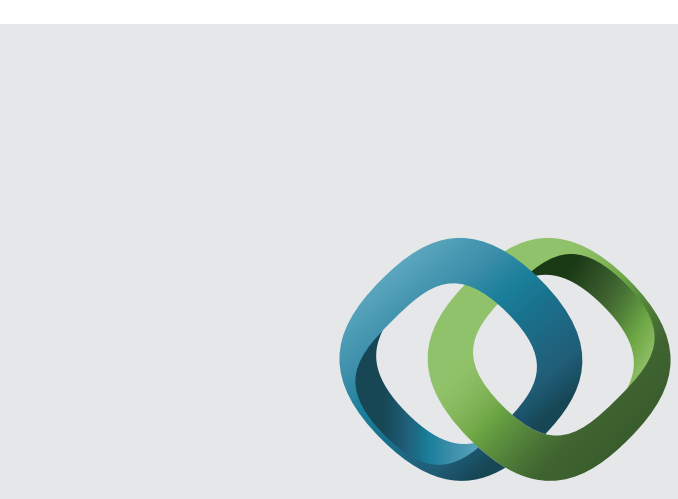

\section{Hindawi}

Submit your manuscripts at

http://www.hindawi.com
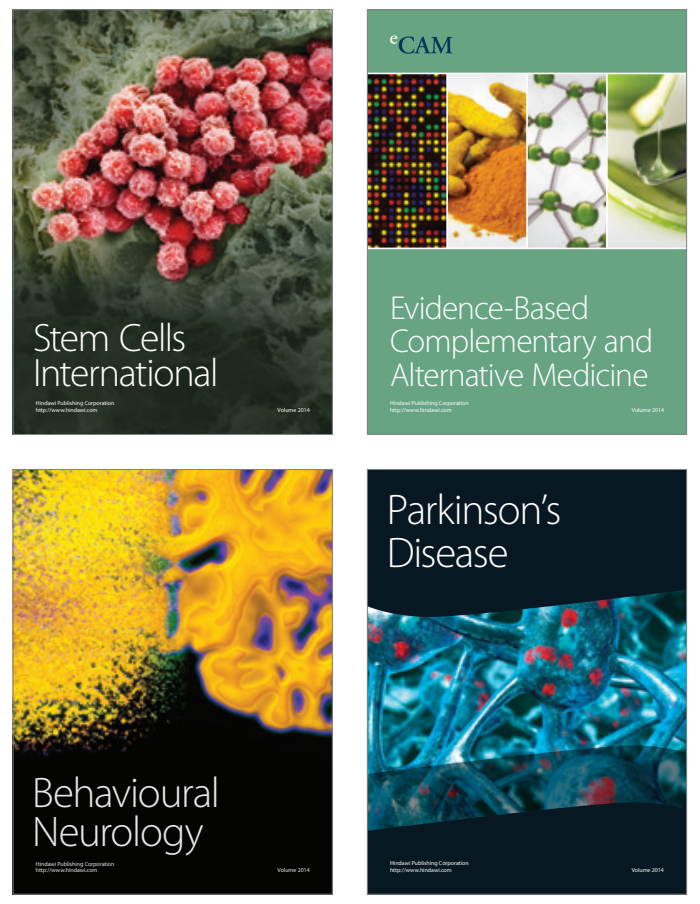
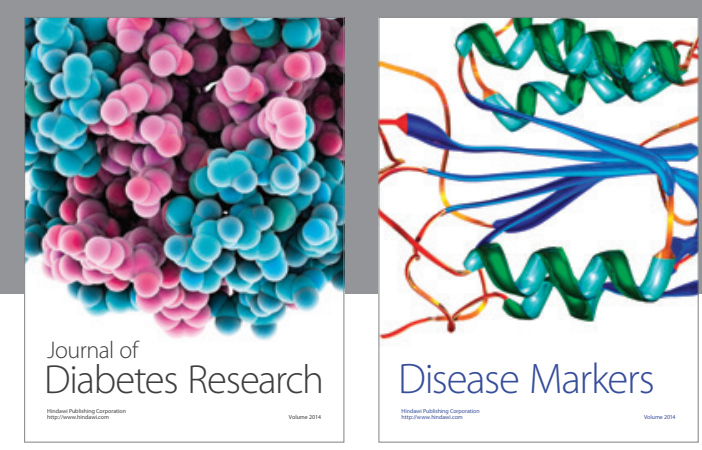

Disease Markers
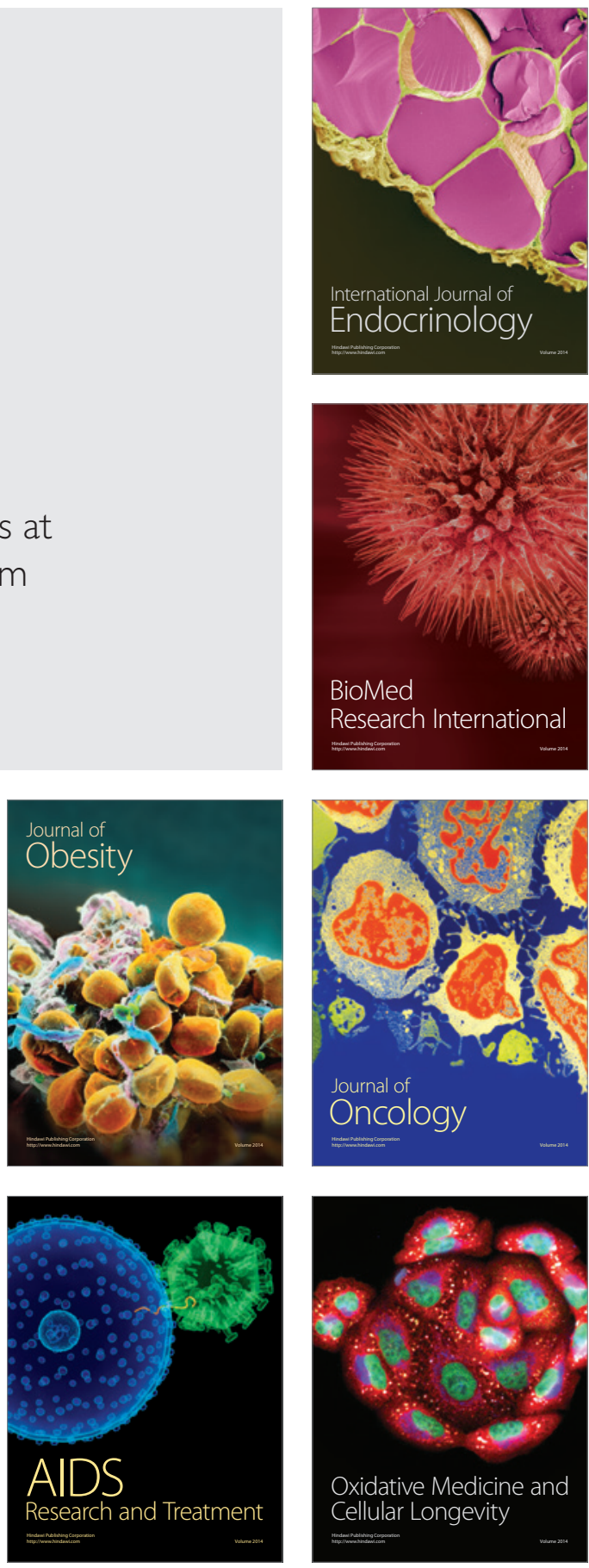